ARTICLE

DOI: $10.1038 / s 41467-018-05775-4$

\title{
Emerging many-body effects in semiconductor artificial graphene with low disorder
}

\author{
Lingjie Du (1) ${ }^{1}$, Sheng Wang ${ }^{1}$, Diego Scarabelli ${ }^{1}$, Loren N. Pfeiffer ${ }^{2}$, Ken W. West ${ }^{2}$, Saeed Fallahi ${ }^{3}$, \\ Geoff C. Gardner ${ }^{3}$, Michael J. Manfra ${ }^{3}$, Vittorio Pellegrini ${ }^{4}$, Shalom J. Wind ${ }^{1}{ }^{1} \&$ Aron Pinczuk ${ }^{1,5}$
}

The interplay between electron-electron interactions and the honeycomb topology is expected to produce exotic quantum phenomena and find applications in advanced devices. Semiconductor-based artificial graphene (AG) is an ideal system for these studies that combines high-mobility electron gases with AG topology. However, to date, low-disorder conditions that reveal the interplay of electron-electron interaction with AG symmetry have not been achieved. Here, we report the creation of low-disorder AG that preserves the nearperfection of the pristine electron layer by fabricating small period triangular antidot lattices on high-quality quantum wells. Resonant inelastic light scattering spectra show collective spin-exciton modes at the M-point's nearly flatband saddle-point singularity in the density of states. The observed Coulomb exchange interaction energies are comparable to the gap of Dirac bands at the $\mathrm{M}$-point, demonstrating interplay between quasiparticle interactions and the AG potential. The saddle-point exciton energies are in the terahertz range, making low-disorder AG suitable for contemporary optoelectronic applications.

\footnotetext{
${ }^{1}$ Department of Applied Physics and Applied Mathematics, Columbia University, New York, NY, USA. ${ }^{2}$ Department of Electrical Engineering, Princeton University, New York, NJ, USA. ${ }^{3}$ Department of Physics and Astronomy, and School of Materials Engineering, and School of Electrical and Computer Engineering, Purdue University, New York, IN, USA. ${ }^{4}$ Istituto Italiano di Tecnologia, Graphene Labs, Via Morego 30, I-16163 Genova, Italy. ${ }^{5}$ Department of Physics, Columbia University, New York, NY, USA. Correspondence and requests for materials should be addressed to L.D. (email: Id2751@columbia.edu)
} 
M any-body effects play important roles in lowdimensional electron systems and attract great deal of attention in graphene physics due to the interplay with the honeycomb topology ${ }^{1-8}$. While theoretical treatments of interaction effects in graphene frequently assume that the systems are clean and controllable, these preconditions are difficult to satisfy in the natural material ${ }^{1-3}$. For instance, the theoretical prediction of chiral superconductivity requires tuning the Fermi energy to the $M$ saddle-points with flatband characteristic ${ }^{2}$, which is difficult in graphene given the large energy gap $(\sim 5 \mathrm{eV}$, a consequence of the small lattice spacing) at the Brillouin-zone (BZ) M-point. Two-dimensional (2D) saddle-point excitons ${ }^{4-7}$ in graphene, redshifted from the saddle-point singularity at the Mpoint, are subject to prominent many-body effects, but their optical responses are at a large energy of $4.6 \mathrm{eV}$, far from the relevant energy ranges for device applications.

Artificial graphene $(A G)^{9-14}$ is a controllable platform for simulation of quantum behavior in the physics of $2 \mathrm{D}$ crystals ${ }^{15-19}$. Linearly dispersing Dirac bands have been reported in several AG systems, including molecular assemblies on copper ${ }^{11}$, fermionic atoms trapped in optical lattices ${ }^{12}$, photonic systems ${ }^{13}$, and nanopatterned GaAs quantum wells (QWs) ${ }^{14}$. AG systems with tunable honeycomb lattices should be suitable for explorations of quantum regimes of many-body effects in graphene-like band structures. Thus far, however, electron-electron interactions in solid-state AG have not been reported, and, particularly in the case of GaAs-based AG, the low-disorder conditions for observing such effects are difficult to achieve.

Here, we report the realization of low-disorder semiconductor AG on a high-mobility GaAs QW. We fabricate small period triangular antidot lattices that significantly suppress the impact of processing disorder on electrons, and thus preserve the high-quality of states in as-grown QWs. The achievement enables observations of collective saddle-point spin excitons that are subject to exchange Coulomb interactions. For AG lattice periods in the range of tens nanometers the energy of the observed saddle-point excitons is in a much sought after terahertz range. Relatively large exchange Coulomb interactions, which have energies comparable to the gap of Dirac bands at the Mpoint of the BZ, could be modulated by tuning the carrier density. The capability of observing collective saddle-point spin excitons and the emergence of relatively large Coulomb interactions in the lowdisorder AG lattices demonstrate access to a regime in AG that is dominated by electron-electron interaction effects, allowing the exploration of intriguing many-body effects that are inaccessible in graphene. Combining Coulomb interactions in a clean electron environment with a meV-energy gap at the $\mathrm{M}$-point provides opportunities for experimental studies of many-body effects in honeycomb lattices such as chiral superconductivity ${ }^{2}$.

\section{Results}

Triangular antidot lattice. We employ state-of-the-art nanofabrication to realize small period triangular antidot lattices in highmobility GaAs QWs. The antidots are created by deep etching of circular holes in the semiconductor in a carefully controlled triangular lattice pattern with period $b$. Figure la shows that the triangular antidot lattice supports a honeycomb lattice of dots (the darker areas in Fig. 1a) with period $a$. The main advantage of this approach is twofold. First, the effective lattice period is smaller than the patterned period by a factor of $\sqrt{3}(a=b / \sqrt{3})$. Second, electrons in the antidot lattice avoid the areas under the etched features due to a large repulsive potential, resulting in a greatly reduced impact of process-induced disorder. This is confirmed in the evaluation of electron density reported in Fig. 1b, which is a characteristic feature of the antidot triangular pattern that results in a $2 \mathrm{D}$ electron gas with physically connected nearest-neighbor honeycomb lattice sites in the unetched areas of the pattern (Fig. 1a, b). Figure 1c displays the calculated dispersion of the two lowest Dirac bands of AG for a typical triangular antidot lattice imprinted on a GaAs QW. The Dirac bands shown in Fig. 1c are gapless with linear momentum dispersion near the $\mathrm{K}\left(\mathrm{K}^{\prime}\right)$ points of the $\mathrm{BZ}$ and with a gap $\left(E_{\mathrm{M}}\right)$ at the $\mathrm{M}$-point that is comparable to the Fermi energy $(\sim 1 \mathrm{meV})$, typical of semiconductor AG.

Device fabrication. The nanofabrication of small period $(60-70 \mathrm{~nm})$ triangular antidot lattices, with periods much smaller than in previous works $(200 \mathrm{~nm})^{20}$, requires precise control of the lattice period, the antidot radius and the deep etching profile. Figure $2 \mathrm{a}-\mathrm{d}$ describes schematically the processing steps involved in the creation of the antidot lattices (see Methods). Electronbeam lithography is employed to define the antidot pattern using a two-step pattern transfer process, that is, based on a Zep 520 electron-beam resist, which possesses both high-resolution capability and good resistance to etching. The e-beam writer is operated at an accelerating voltage of $80 \mathrm{kV}$ to mitigate proximity effects that are relatively strong in $\mathrm{GaAs}^{21-23}$. A temperature-
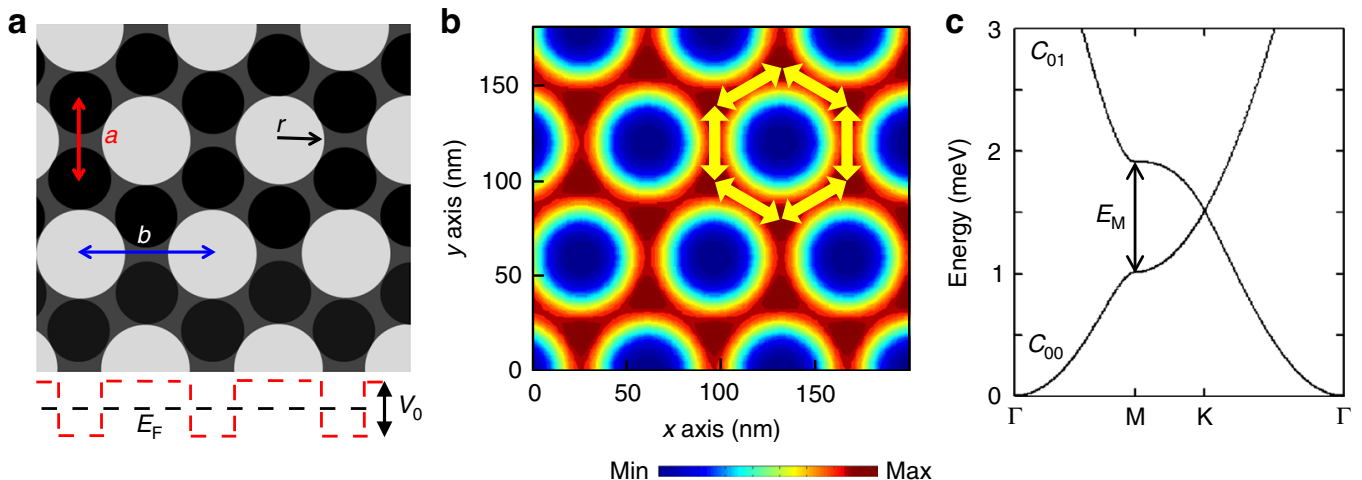

Fig. 1 Low-disorder artificial graphene in triangular antidot lattices. a Schematic of a triangular antidot lattice with period $b$ that is imprinted on a GaAs QW. The white circles represent etched antidots of radius $r$. The black and gray areas represent the unetched areas, and the black circles indicate the effective dots arranged in a honeycomb pattern of period $a=b / \sqrt{3}$. In the evaluation of AG band structure, we used a muffin-tin potential (red dashed line) with Fermi energy $E_{\mathrm{F}}$ in the single-particle approximation $9,10,14 . V_{\mathrm{O}}$ is the potential depth in the etched area. Each antidot produces an effectively repulsive potential $V_{0}$. b Evaluations of electron density in a triangular antidot lattice with $V_{0}=6 \mathrm{meV}, b=70 \mathrm{~nm}, a=40.4 \mathrm{~nm}$, and $r=20 \mathrm{~nm}$. Yellow arrows highlight strengthened electron coupling between nearest-neighbor dots. The color bar indicates the electron density. c The two lowest Dirac bands of AG with the parameters in $\mathbf{b}$. $E_{M}$ indicates the value of the gap near the $M$-point 

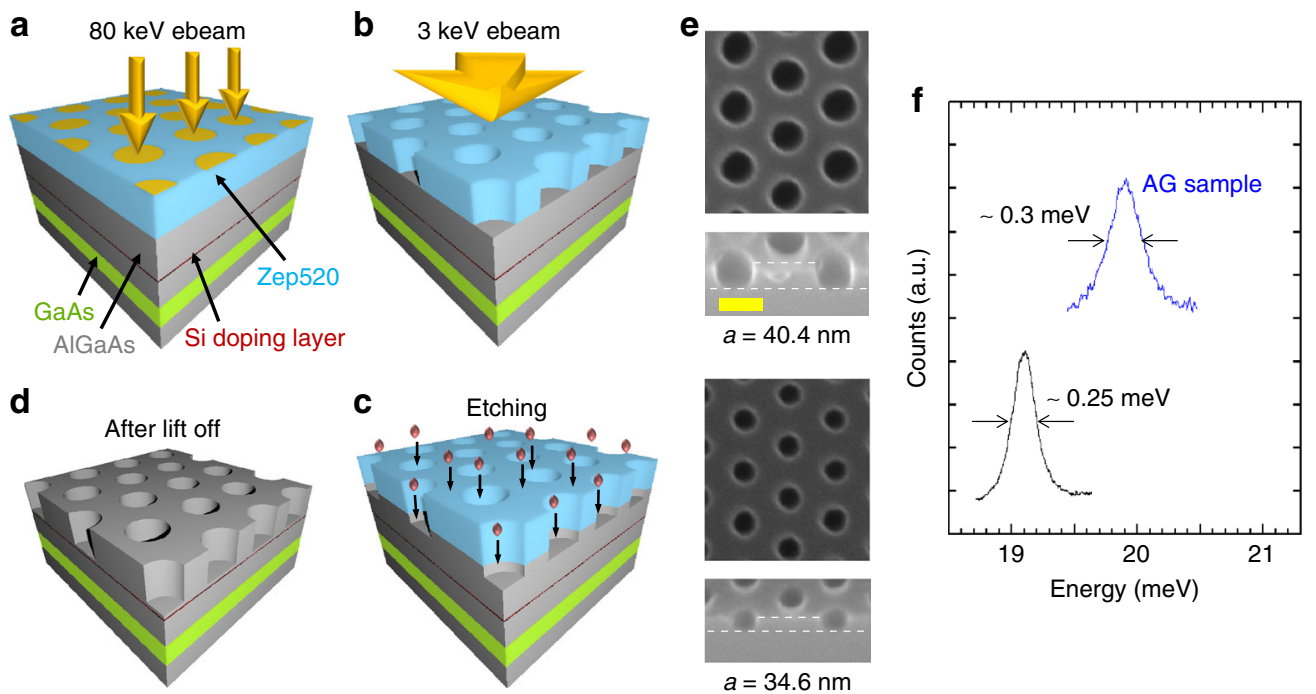

Fig. 2 Creation of artificial graphene in triangular antidot lattices. Processing steps: a a Zep 520 resist is exposed by an electron beam at $80 \mathrm{kV}$ accelerating voltage. After exposure, the development of the resist produces triangular antidots pattern. $\mathbf{b}$ The resist with pattern is exposed by an electron flood at $3 \mathrm{kV}$ accelerating voltage, to enhance the chemical stability of the resist. $\mathbf{c}$ The $\mathrm{BCl}_{3}$-based dry etch is performed to transfer the pattern to the substrate with depth control. The final device after the removal of the residue resist is shown in $\mathbf{d}$. The $\mathrm{QW}$ is positioned $80 \mathrm{~nm}$ below the surface and $30 \mathrm{~nm}$ below the $\mathrm{Si} \delta$-doping layer. The as-grown electron density is $2.1 \times 10^{11} \mathrm{~cm}^{-2}$, with a Fermi energy $E_{\mathrm{F}}$ of $7.5 \mathrm{meV}$ and a typical low-temperature mobility of $10^{6} \mathrm{~cm}^{2} \mathrm{~V}^{-1} \mathrm{~s}^{-1}$. e Scanning electron microscopy micrographs of AG lattices with different periods in $0^{\circ}$ tilt (upper panel, top view) and $40^{\circ}$ tilt (lower panel, side view). The white dashed lines define the etching depth. The yellow scale bar is $50 \mathrm{~nm}$ for all panels in $\mathbf{e}$. $\mathbf{f}$ The comparison between the RILS spectrum of intersubband excitations of AG sample I (with incident photon energy of $1554.36 \mathrm{meV}$, blue trace) and intersubband excitations of the as-grown GaAs QW (with incident photon energy of $1550.92 \mathrm{meV}$, black trace). The peak width of intersubband excitations of sample I is nearly constant for different incident photon energies (see Supplementary Note 5 and Supplementary Figure 8). The spectra were taken at $5 \mathrm{~K}$ under crossed polarization

controlled, modified developing process with ultrasonication is applied (see Methods), followed by a low voltage $(\sim 3 \mathrm{kV})$ blanket electron-beam exposure to harden the resist ${ }^{24}$. This is followed by a $\mathrm{BCl}_{3}$-based inductively coupled plasma reactive-ion etching step designed to transfer the triangular antidot array to the QW. Arrays with lattice constants $b$ as small as $60 \mathrm{~nm}$ are fabricated over an area of $200 \times 200 \mu \mathrm{m}^{2}$. Figure $2 \mathrm{e}$ shows top and side views of samples having patterns with $b=60 \mathrm{~nm}(a=34.6 \mathrm{~nm})$ and $b=70 \mathrm{~nm}(a=40.4 \mathrm{~nm})$ (sample parameters are listed in Supplementary Table 1). The structures are characterized by photoluminescence to determine the Fermi energy (Supplementary Figure 1). Photoluminescence outside the AG pattern where no mask is present shows that the etching depth in the sample with $a=40.4 \mathrm{~nm}$ (sample I) leads to a full depletion of electrons, suggesting that electron wavefunctions are strongly suppressed under the antidots. The estimated potential barrier is $V_{0}=6 \mathrm{meV}$ (see Supplementary Note 1).

Low-disorder AG. The impact of disorder resulting from nanofabrication processing is assessed by resonant inelastic light scattering (RILS) ${ }^{14,19}$ spectra of intersubband collective excitations ${ }^{25}$, in which the only electron degree of freedom that changes is the confinement state in the QW. In Fig. $2 \mathrm{f}$, a comparison between intersubband excitations of sample I and the as-grown QW under crossed incident and scattered photon polarizations shows that the peak in AG has a full width at half maximum (FWHM) of $0.3 \mathrm{meV}$, which is extremely close to the FWHM $\sim 0.25 \mathrm{meV}$ of the mode in the as-grown QW (the FWHM reflects the degree of disorder in the device, which is typically due to ionized defects that scatter electrons ${ }^{26}$ ). The slight increase of FWHM could be attributed to the reduced screening of potential of ionized defects due to the greatly reduced Fermi energy (reduced charge density) in the AG sample (see Supplementary Table 1 and Supplementary Note 1). The nearly identical width of the modes in AG to that in the as-grown QW indicates the exceptional low-disorder environment for charge carriers in this small period triangular antidot lattice. This is in contrast with results from a honeycomb dot lattice with optimized fabrication processing and similar period under crossed polarization, where the FWHM increases by a factor of three from the as-grown QW value $^{25}$.

Low-energy Dirac-band transitions. The AG band structure in sample I is probed by RILS measurements of low-energy excitations from Dirac-band transitions shown in Fig. 3a. RILS spectra under crossed polarization such as those in Fig. 3b, c access excitations in the spin degree of freedom ${ }^{27}$. Spin-density excitations involving changes in the spin degrees of freedom are redshifted from single-particle transitions due to exchange Coulomb interactions. This is an excitonic shift due to Coulomb coupling between the excited electrons and the holes in the lower state 28,29 . Charge density excitations that preserve spin orientation are active in parallel polarization where the laser tail becomes strong and obscures RILS signals in low-energy excitations. Spectra of low-energy excitations presented here are under cross polarization.

In the absence of many-body interaction effects the RILS spectra are proportional to the joint density of states (JDOS) for the transitions ${ }^{14}$. The two lower curves in Fig. $3 \mathrm{~b}$ are the calculated JDOS from the parameters in Fig. 3a for sample I with $\gamma=0 \mathrm{meV}$ and $\gamma=0.1 \mathrm{meV}$ ( $\gamma$ is Gaussian broadening width). The evaluations show that the JDOS has a maximum at $E_{\mathrm{M}}=0.9$ $\mathrm{meV}(0.2 \mathrm{THz})$ arising from the saddle-point singularity for transitions at the M-point of the BZ (cyan areas in Fig. 3a, b). The rapid collapse of the RILS intensity at energies below $E_{\mathrm{M}}$, seen in the top curve in Fig. $3 \mathrm{~b}$, is a signature of the formation of linearly dispersing Dirac cones ${ }^{14}$, thus confirming the presence of a Dirac-band structure in the triangular antidot lattice in sample I. 

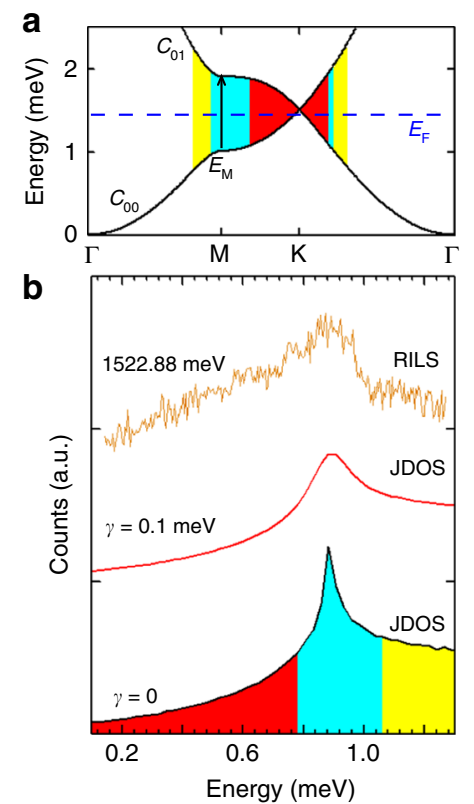
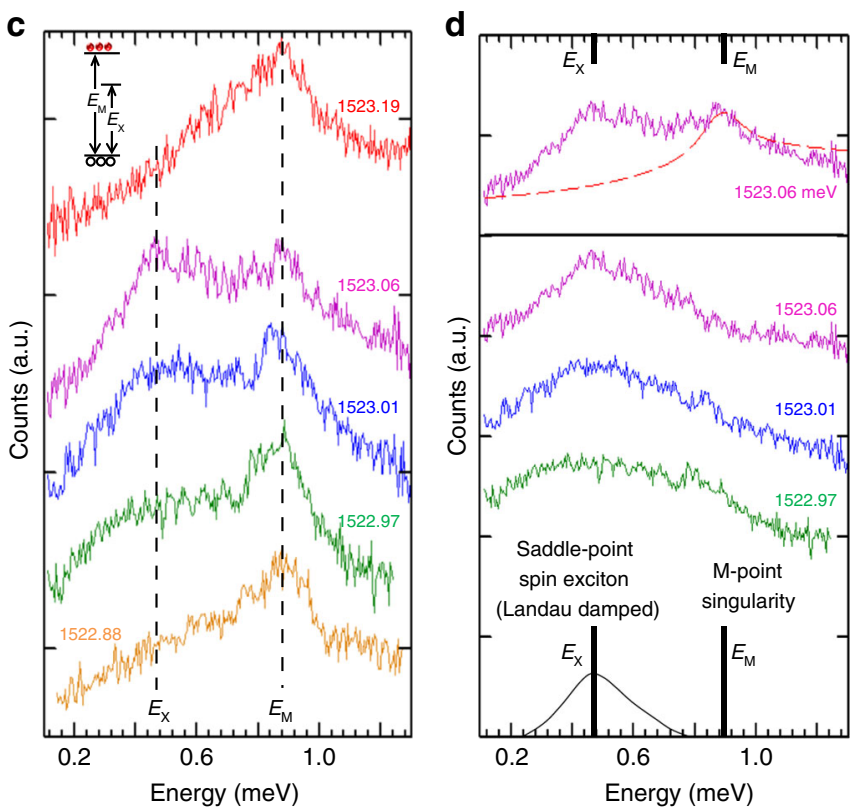

Fig. 3 Dirac-band excitations in low-disorder AG in a triangular antidot structure. a AG energy band calculation with parameters in Supplementary Table 1 and diagram of Dirac-band transitions for sample I. Transitions in different regimes are illustrated by different colors. $\mathbf{b}$ The lowest plot is the joint density of states (JDOS) for the transitions in $\mathbf{a}$. The colored areas show transitions in different regimes. The red trace is the JDOS under Gaussian broadening with width $\gamma=0.1 \mathrm{meV}$ (this $\gamma$ is chosen to fit the spectra line shape near $E_{M}$ ). The top yellow trace is the RILS spectrum of Dirac-band excitations in sample I obtained with incident photon energy $\hbar \omega_{i}$ of $1522.88 \mathrm{meV}$. c RILS spectra of Dirac-band excitations. A non-RILS background has been removed (subtraction details are reported in Supplementary Figures 4-6, and in Supplementary Note 3). $\hbar \omega_{i}$ in meV are indicated. The vertical dashed lines indicate the positions of $E_{\mathrm{M}}$ and $E_{\mathrm{X}}$. The inset shows a level diagram for $E_{\mathrm{M}}$ and $E_{\mathrm{X}}$. In the inset, black circles are for holes in $c_{00}$ band and red circles are for electrons in $c_{01}$ band. d Top shows a RILS spectrum (purple trace) with $\hbar \omega_{i}$ of $1523.06 \mathrm{meV}$, and the JDOS (red dashed line, $\gamma=0.1$ meV) that is proportional to scattering intensity due to the single-particle transitions. Bottom: upper traces reveal the spectrum after subtraction of the single-particle JDOS intensities with $\hbar \omega_{\mathrm{i}}$ of $1523.06 \mathrm{meV}$ (purple), $1523.01 \mathrm{meV}$ (blue), and $1522.97 \mathrm{meV}$ (green). A schematic plot shows the spin-exciton energy and the gap energy at the M-point. Due to Landau damping, the width of the spin-exciton mode increases

The RILS spectrum in Fig. 3b also uncovers departures from the calculated single-particle JDOS at energies just below $E_{\mathrm{M}}$. The resonance behavior of RILS spectra shown in Fig. $3 \mathrm{c}$ reveals that the spectral lineshapes of low-energy Dirac-band excitations have a strong dependence on incident photon energy $\hbar \omega_{\mathrm{i}}$ (see Supplementary Note 7). Major departures of the RILS spectral lineshapes from the JDOS predictions for energies below $E_{\mathrm{M}}$ can be seen in spectra in Fig. 3d, where the single-particle JDOS has been subtracted from the spectra in Fig. 3c, revealing a relatively broad intensity maximum at $E_{\mathrm{X}}$. The redshift from $E_{\mathrm{M}}$ is interpreted as arising from many-body electron interactions that emerge in the low-disorder AG. For spin excitations in Fig. 3 the relevant manybody effects emanate from exchange Coulomb interactions. Then, it is assigned to collective spin excitations associated with the saddlepoint singularity for Dirac-band transitions. An alternative interpretation for the $E_{\mathrm{X}}$ mode is discussed in Supplementary Note 6 and Supplementary Figure 9. The energy of saddle-point excitons $E_{\mathrm{X}}$ associated with transitions at the M-point singularity would be at an energy of about $0.45 \mathrm{meV}(0.1 \mathrm{THz})$, and tunable (e.g., by changing the lattice constant).

The significant broadening of the $E_{\mathrm{X}}$ maximum (in the spectra of Fig. 3d) is due to overlap with the continuum of singleparticle excitations, which causes the decay of the spin-exciton mode into electron-hole pairs (Landau damping ${ }^{29}$, see Fig. $3 \mathrm{~d}$ ); this is also observed at the saddle-point exciton in natural graphene ${ }^{5-7}$. An exchange Coulomb energy in the saddle-point exciton here can be estimated as in the case of intersubband excitations: ${ }^{29}$ $\left(E_{M}^{2}-E_{X}^{2}\right) / E_{\mathrm{M}}=0.6 \mathrm{meV}$. This is a relatively large exchange interaction strength that is about half of the M-point gap $E_{\mathrm{M}}$. As shown in ref. ${ }^{29}$, exchange Coulomb energies are equal to $E_{\mathrm{ex}}=2 n \beta$, where $n$ is the carrier density and $\beta$ is the exchange interaction matrix element for the transition. Here, the carrier density in the $c_{00}$ band is linked to the exchange energy in low-energy excitations $\left(c_{00} \rightarrow c_{01}\right)$. Exchange energies in the same range could be expected in other transitions associated to the carrier density in the $c_{00}$ band.

Spin-density intersubband excitations. A large exchange interaction is observed in the spin-density intersubband excitation (SDE) shown in Fig. 4, in which there is no change in the states of the AG potential (intersubband excitations under parallel polarization are presented in Supplementary Figures 7 and 8, and in Supplementary Note 4). The exchange energy associated with $\mathrm{SDE}$ can be written as $E_{\mathrm{ex}}=\left(E_{10}^{2}-E_{\mathrm{SDE}}^{2}\right) / E_{10}{ }^{29}$, where $E_{\mathrm{SDE}}$ is the peak position of SDE and $E_{10}=20.0 \mathrm{meV}$ is the QW subband spacing (see Supplementary Note 2, Supplementary Figures 2 and 3). Figure 4a displays RILS processes for intersubband transitions that are from $c_{00}\left(c_{01}\right)$ band to $c_{10}\left(c_{11}\right)$ band in the second QW subband. As shown in Fig. $4 \mathrm{a}, c_{00} \rightarrow c_{10}$ transitions have a resonance enhancement at incident photon energies $\hbar \omega_{\mathrm{i}}$ that are lower than the resonance for $c_{01} \rightarrow c_{11}$ transitions. Figure $4 \mathrm{~b}$ shows a color plot of the resonance enhancement for SDE modes at around $20 \mathrm{meV}$ that reveals a remarkable dependence of peak positions on $\hbar \omega_{\mathrm{i}}$. This can be explained by the different resonance enhancements in RILS by excitations associated with different transitions. At lower $\hbar \omega_{\mathrm{i}}$ the SDE spectra come from excitations linked to $c_{00} \rightarrow c_{10}$ transitions, whilst at higher $\hbar \omega_{\mathrm{i}}$ the spectra are largely due to excitations from $c_{01} \rightarrow c_{11}$ transitions. The changes in the position of SDE peaks as a function of $\hbar \omega_{\mathrm{i}}$ in Fig. $4 \mathrm{~b}$ are attributed to the different exchange interactions in those two transitions. For a low $\hbar \omega_{\mathrm{i}}=1552.5 \mathrm{meV}$ the SDE mode is at $E_{\mathrm{SDE}}$ $=19.8 \mathrm{meV}$ and the exchange energy is $E_{\mathrm{ex}}=0.43 \pm 0.05 \mathrm{meV}$ for 

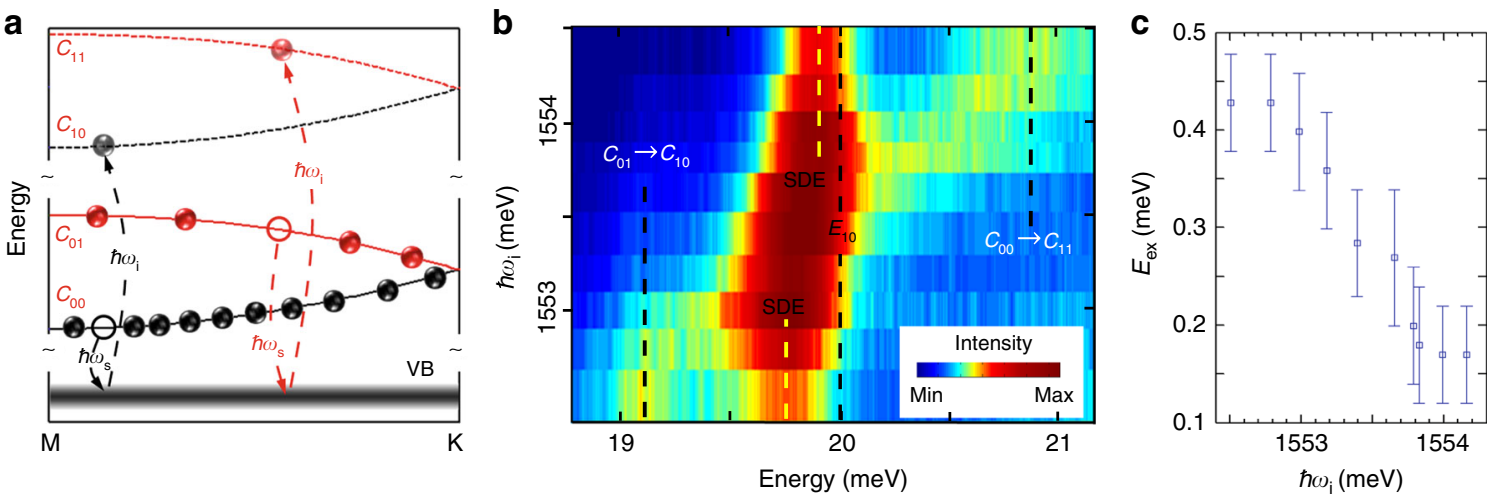

Fig. 4 Coulomb exchange energy probed in intersubband excitations. a Optical transitions in RILS of intersubband spin-density excitations (SDE) are indicated. In transitions with incident photon energy $\hbar \omega_{\mathrm{i}}$, the incident photon excites an electron from the valence band (VB) to the $c_{11} / c_{10}$ state leaving behind a hole. In transitions with scattered photon energy $\hbar \omega_{s^{\prime}}$ an electron in the $c_{01} / c_{00}$ state recombines with the hole and emits the scattered photon. The incident photon energy $\hbar \omega_{\mathrm{i}}$ and scattered photon energies $\hbar \omega_{\mathrm{s}}$ are linked by energy conservation $E=\hbar \omega_{\mathrm{i}}-\hbar \omega_{\mathrm{s}}$, where $E$ is the energy of $A G$ excitations being studied. In SDE, the electrons are excited from a lower populated AG subband to the next higher subband without the change in AG band index. Red (black) line defines SDE exciting electron states with spin-flip processing from $c_{01}$ and $c_{11}$ (from $c_{00}$ and $c_{10}$ ) under higher (lower) $\hbar \omega_{j}$. While the $c_{01}$ band is above the Fermi energy it is populated because of the thermal excitation of charge carriers at finite temperature of our experiments. $\mathbf{b}$ Color plot of RILS spectra as a function of $\hbar \omega_{\mathrm{i}}$ measured at temperature $T=5 \mathrm{~K}$ with crossed polarization. The color bar indicates intensities of scattered light. The dashed lines marked with $c_{01} \rightarrow c_{10}$ and $c_{00} \rightarrow c_{11}$ indicate the energies of combined intersubband transitions with the change in both quantum well subband and $A G$ band index (see ref. ${ }^{25}$ and Supplementary Note 2). The dashed line marked with $E_{10}$ indicates the position of quantum well subband spacing (see Supplementary Note 2). The yellow dashed lines indicate the position of SDE. There is a weak photoluminescence that overlaps the $c_{01} \rightarrow c_{10}$ and $c_{00} \rightarrow c_{11}$ transitions, as well as SDE. c The dependence of exchange energies extracted from (b) on $\hbar \omega_{\mathrm{i}}$. The vertical error bars represent estimated uncertainties in determination of $E_{10}$ and $E_{\mathrm{SDE}}$ from the measured spectra

$c_{00} \rightarrow c_{10}$ transitions. The large exchange interaction confirms the results obtained in the saddle-point spin-exciton mode. For a high $\hbar \omega_{\mathrm{i}}=1554.0 \mathrm{meV}$ the SDE mode is at $E_{\mathrm{SDE}}=19.9 \mathrm{meV}$ and the exchange energy is $E_{\mathrm{ex}}=0.17 \pm 0.05 \mathrm{meV}$ for $c_{01} \rightarrow c_{11}$ transitions. The dependence of $E_{\mathrm{ex}}$ on $\hbar \omega_{\mathrm{i}}$ is shown in Fig. 4c.

The much smaller exchange energy for $c_{01} \rightarrow c_{11}$ transitions is attributed to lower carrier population in the $c_{01}$ band of $A G$, since exchange Coulomb energies are proportional to carrier densities. While the $c_{01}$ band is above the Fermi energy it is populated because of the thermal excitation of charge carriers at finite temperature of our experiments. Data points in Fig. $4 \mathrm{c}$ suggest $E_{\mathrm{ex}}$ $\left(c_{00} \rightarrow c_{10}\right) / E_{\text {ex }} \quad\left(c_{01} \rightarrow c_{11}\right) \sim 3$. According to the Fermi-Dirac distribution at the temperature $5 \mathrm{~K}$, we estimate $n_{c 00} / n_{c 01} \sim 3$, where $n_{c 00}$ and $n_{c 01}$ are carrier densities in the $c_{00}$ and $c_{01}$ bands, showing $E_{\mathrm{ex}}\left(c_{00} \rightarrow c_{10}\right) / E_{\mathrm{ex}}\left(c_{01} \rightarrow c_{11}\right) \sim n_{c 00} / n_{c 01} \sim 3$. Furthermore, as shown in Supplementary Note 8 and in Supplementary Figures 11-13, sample III has a higher Fermi energy, so that $n_{c 00}$ and $n_{c 01}$ are very close. Then, the determined exchange energies for $c_{01} \rightarrow c_{11}$ and $c_{00} \rightarrow c_{10}$ transitions, with similar densities, are comparable. The tuning of $E_{\text {ex }}$ by the electron density provides an effective way to control saddle-point spin-exciton modes and for future studies of excitonic ground states.

Exchange interactions in low-energy excitations $\left(c_{00} \rightarrow c_{01}\right)$ and $\operatorname{SDE}\left(c_{00} \rightarrow c_{10}\right)$ are linked to the carrier density in the $c_{00}$ band, determining similar exchange energies in these excitations. On the other hand, transitions in $c_{00} \rightarrow c_{01}$ and $c_{00} \rightarrow c_{10}$ have different values of matrix elements $\beta$, which would result the difference between the exchange energies $\left(0.6 \mathrm{meV}\right.$ in $c_{00} \rightarrow c_{01}$ transitions and $0.45 \mathrm{meV}$ in $c_{00} \rightarrow c_{10}$ transitions). At a carrier density of $0.4 \times 10^{11} \mathrm{~cm}^{-2}$ in the $c_{00}$ band, we have $\beta \approx 0.7 \times 10$ $-11 \mathrm{~cm}^{2} \mathrm{meV}^{-1}$ for the low-energy excitations based on $c_{00} \rightarrow c_{01}$ transitions, and $\beta \approx 0.5 \times 10^{-11} \mathrm{~cm}^{2} \mathrm{meV}^{-1}$ for the SDE based on $c_{00} \rightarrow c_{01}$ transitions. It is known that exchange Coulomb interactions in as-grown GaAs QW are strong due to large values of $\beta^{29}$. Values of $\beta$ we obtained in the AG sample are in the same range as the as-grown samples $\left(0.8 \times 10^{-11} \mathrm{~cm}^{2} \mathrm{meV}^{-1}\right.$ measured in ref. $\left.{ }^{29}\right)$, resulting in large exchange interactions.

\section{Discussion}

The realization of low-disorder AG in small period triangular antidot lattices, which reveals the presence of collective terahertz saddle-point spin excitons at the M-point singularity in the density of states. We observe relatively large exchange Coulomb interactions, which have energies comparable to the gap of Dirac bands at the M-point. Low-disorder AG is thus a highly tunable condensedmatter system for the investigation of graphene-like physics in a regime where the interplay between electron interactions and the hexagonal topology of AG lattices becomes predominant. The large exchange coupling to the honeycomb lattice with tunable parameters makes low-disorder AG lattice potentially suitable for further studies of exciton liquids and spintronics ${ }^{30}$. To realize possible exciton liquids ${ }^{31,32}$, the suppression of Landau damping is required, which would be achieved by opening a gap at $K\left(K^{\prime}\right)$ points (through removing inversion symmetry of the AG lattice). Our findings may open approaches for the investigation of strongly correlated quantum phases in condensed-matter systems such as ferromagnetism and unconventional superconductivity ${ }^{2,3}$, expanding the tool box for quantum simulation. The low-disorder processing approach can be applied to artificial lattices with intriguing topologies such as the Lieb ${ }^{33,34}$ and Kagome lattices. Given that the saddle-point excitons are in the terahertz frequency range and subject to tunable parameters, low-disorder AG may provide a semiconductor platform for optoelectronic device applications.

\section{Methods}

Fabrication of the AG nanopattern. The semiconductor system we used is 25-nm-wide one-side modulation-doped $\mathrm{Al}_{0.1} \mathrm{Ga}_{0.9} \mathrm{As} / \mathrm{GaAs} \mathrm{QW}$. The $\mathrm{QW}$ is positioned $80 \mathrm{~nm}$ below the surface and $30 \mathrm{~nm}$ below the Si $\delta$-doping layer. The asgrown electron density is $2.1 \times 10^{11} \mathrm{~cm}^{-2}$, with a Fermi energy $E_{\mathrm{F}}$ of $7.5 \mathrm{meV}$ and a typical low-temperature mobility of $10^{6} \mathrm{~cm}^{2} \mathrm{~V}^{-1} \mathrm{~s}^{-1}$. Electron-beam lithography at $80 \mathrm{kV}$ and beam current $400 \mathrm{pA}$ (e-beam writer nB4, NanoBeam Ltd.) was employed to pattern $200 \times 200 \mu \mathrm{m}^{2}$ triangular arrays of circles. We used a single layer resist of Zep 520 as a hard mask, which was developed in a solution of n-Amyl acetate: isopropanol in composition 1:3 with ultrasound sonication. In the development, the temperature is always kept at $0^{\circ}$. After development, a $3 \mathrm{kV}$ electron beam was flood exposed on the pattern to harden the developed resist. An Oxford PlasmaPro System 100 was used to perform the inductively coupled plasma 
reactive-ion etching. The gas employed was a mixture of $50 \mathrm{sccm}$ Ar and $10 \mathrm{sccm}$ $\mathrm{BCl}_{3}$, shown to etch AlGaAs/GaAs heterostructures with high anisotropy with the etching time $60 \mathrm{~s}$. The reactive-ion etching power and ion coupled plasma power were 45 and $200 \mathrm{~W}$ with a pressure of 8 mtorr. The residual resist was removed with dimethylacetamide. The etch recipe was optimized to achieve a high degree of physical bombardment and reduced chemical etching.

Optical measurements. The sample was mounted in an optical cryostat with a base temperature of $4 \mathrm{~K}$. The RILS experiments were performed in a back-scattering configuration, with incident laser beam almost perpendicular (within $10^{\circ}$ ) to the sample. A tunable Ti:sapphire laser with spot diameter of around $200 \mu \mathrm{m}$ and power as low as $20 \mu \mathrm{W}$ was focused on the AG nanopattern. Spectra were collected using a liquid nitrogen cooled CCD through a double grating spectrometer (Spex 1404).

Data availability. The authors declare that the data that support the plots within this paper and other findings of this study are available from the corresponding author upon reasonable request.

Received: 13 March 2018 Accepted: 18 July 2018

Published online: 17 August 2018

\section{References}

1. Kotov, V. N., Uchoa, B., Pereira, V. M., Guinea, F. \& Neto, A. H. C. Electron-electron interactions in graphene: current status and perspectives. Rev. Mod. Phys. 84, 1067 (2012).

2. Nandkishore, R., Levitov, L. S. \& Chubukov, A. V. Chiral superconductivity from repulsive interactions in doped graphene. Nat. Phys. 8, 158 (2012).

3. Meng, Z. Y., Lang, T. C., Wessel, S., Assaad, F. F. \& Muramatsu, A. Quantum spin liquid emerging in two-dimensional correlated Dirac fermions. Nature 464, 847 (2010).

4. Yang, L., Deslippe, J., Park, C. H., Cohen, M. L. \& Louie, S. G. Excitonic effects on the optical response of graphene and bilayer graphene. Phys. Rev. Lett. 103, 186802 (2009)

5. Kravets, V. G. et al. Spectroscopic ellipsometry of graphene and an excitonshifted van Hove peak in absorption. Phys. Rev. B 81, 155413 (2010).

6. Mak, K. F., Shan, J. \& Heinz, T. F. Seeing many-body effects in single- and few-layer graphene: observation of two-dimensional saddle-point excitons. Phys. Rev. Lett. 106, 046401 (2011).

7. Chae, D. H. et al. Excitonic Fano resonance in free-standing graphene. Nano Lett. 11, 1379-1382 (2011).

8. Basov, D. N., Fogler, M. M., Lanzara, A., Wang, F. \& Zhang, Y. Colloquium: graphene spectroscopy. Rev. Mod. Phys. 86, 959 (2014).

9. Park, C. H. \& Louie, S. G. Making massless Dirac Fermions from a patterned two-dimensional electron gas. Nano Lett. 9, 1793 (2009).

10. Gibertini, M. et al. Engineering artificial graphene in a two-dimensional electron gas. Phys. Rev. B 79, 241406 (2009).

11. Gomes, K. K., Mar, W., Ko, W., Guinea, F. \& Manoharan, H. C. Designer Dirac fermions and topological phases in molecular graphene. Nature 483, 306 (2012).

12. Tarruell, L., Greif, D., Uehlinger, T., Jotzu, G. \& Esslinger, T. Creating, moving and merging Dirac points with a Fermi gas in a tunable honeycomb lattice. Nature 483, 302 (2012).

13. Jacqmin, T. et al. Direct observation of Dirac cones and a flatband in a honeycomb lattice for polaritons. Phys. Rev. Lett. 112, 116402 (2014).

14. Wang, S. et al. Observation of Dirac bands in artificial graphene in small period nano-patterned GaAs quantum wells. Nat. Nanotech. 13, 29-33 (2017).

15. Novoselov, K. S. et al. Two-dimensional gas of massless Dirac fermions in graphene. Nature 438, 197 (2005).

16. Zhang, Y. B., Tan, Y. W., Stormer, H. L. \& Kim, P. Experimental observation of the quantum Hall effect and Berry's phase in graphene. Nature 438, 201 (2005).

17. Neto, A. H. C., Guinea, F., Peres, N. M. R., Novoselov, K. S. \& Geim, A. K. The electronic properties of graphene. Rev. Mod. Phys. 81, 109 (2009).

18. Polini, M., Guinea, F., Lewenstein, M., Manoharan, H. C. \& Pellegrini, V. Artificial honeycomb lattices for electrons, atoms and photons with honeycomb topology. Nat. Nanotech. 8, 625 (2013).

19. Singha, A. et al. Two-dimensional Mott-Hubbard electrons in an artificial honeycomb lattice. Science 332, 1176 (2011).

20. Nadvornik, L. et al. From laterally modulated two-dimensional electron gas toward artificial graphene. New J. Phys. 14, 053002 (2012).

21. Scarabelli, D. et al. Fabrication of artificial graphene in a GaAs quantum heterostructure. J. Vac. Sci. Technol. B 33, 06FG03 (2015).

22. Czaplewski, D. A., Holt, M. V. \& Ocola, L. E. The range and intensity of backscattered electrons for use in the creation of high fidelity electron-beam lithography patterns. Nanotechnology 24, 305302 (2013).
23. Shimizu, R. \& Ding, Z. J. Monte-Carlo modeling of electron-solid interactions Rep. Prog. Phys. 55, 487 (1992)

24. Czaplewski, D. A. \& Ocola, L. E. Increased pattern transfer fidelity of ZEP $520 \mathrm{~A}$ during reactive-ion etching through chemical modifications by additional dosing of the electron-beam resist. J. Vac. Sci. Technol. B 29, 021601 (2011).

25. Wang, S. et al. Observation of electron states of small period artificial graphene in nano-patterned GaAs quantum wells. Appl. Phys. Lett. 109, 113101 (2016).

26. Pinczuk, A., Worlock, J. M., Stormer, H. L., Gossard, A. C. \& Wiegmann, W Light scattering spectroscopy of electrons in GaAs-(AlGa)As heterostructures: correlation with transport properties. J. Vac. Sci. Technol. 19, 561-563 (1981).

27. Danan, G. et al. Coupling of excitons with free electrons in light scattering from GaAs quantum wells. Phys. Rev. B 39, 5512-5515 (1989).

28. Katayama, S. \& Ando, T. Light scattering by electronic excitations in n-type GaAs-Alx ${ }_{G} \mathrm{a}_{1-\mathrm{x} A}$ s superlattices. J. Phys. Soc. Jpn. 54, 1615-1626 (1985).

29. Pinczuk, A. et al. Large exchange interactions in the electron gas of GaAs quantum wells. Phys. Rev. Lett. 63, 1633-1636 (1989).

30. Han, W., Kawakami, R., Gmitra, M. \& Fabian, J. Graphene spintronics. Nat. Nanotech. 9, 794 (2014)

31. Keldysh, L. V. K. \& Kopaev, Y. V. Possible instability of the semimetallic state toward coulomb interaction. Fiz. Tverd. Tela 6, 2791-2798 (1964).

32. Jérome, D., Rice, T. M. \& Kohn, W. Excitonic insulator. Phys. Rev. 158, 462-475 (1967)

33. Drost, R., Ojanen, T., Harju, A. \& Liljeroth, P. Topological states in engineered atomic lattices. Nat. Phys. 13, 668-671 (2017).

34. Slot, M. R. et al. Experimental realization and characterization of an electronic Lieb lattice. Nat. Phys. 13, 672-676 (2017).

\section{Acknowledgments}

The work at Columbia University was supported by grant DE-SC0010695 funded by the US Department of Energy Office of Science, Division of Materials Sciences and Engineering; and by the National Science Foundation, Division of Materials Research under award DMR-1306976. The growth of GaAs/AlGaAs QWs at Purdue University was supported by grant DE-SC0006671 funded by the US Department of Energy Office of Science, Division of Materials Sciences and Engineering. The work at Princeton University was funded by the Gordon and Betty Moore Foundation through the EPiQS initiative Grant GBMF4420, and by the National Science Foundation MRSEC Grant DMR 1420541. V.P. acknowledges funding from the European Union's Horizon 2020 research and innovation program under grant agreement No. 696656GrapheneCore1.

\section{Author contributions}

L.D. fabricated the AG lattices and performed optical experiments; L.D. and S.W. performed numerical calculations; L.D. and A.P. analyzed the data; S.W. and D.S. contributed to initial experiments; G.C.G., S.F., M.J.M., K.W., and L.N.P. fabricated the QW samples; L.D., A.P., S.J.W., and V.P. cowrote the paper with input from other authors

\section{Additional information}

Supplementary Information accompanies this paper at https://doi.org/10.1038/s41467018-05775-4.

Competing interests: The authors declare no competing interests.

Reprints and permission information is available online at http://npg.nature.com/ reprintsandpermissions/

Publisher's note: Springer Nature remains neutral with regard to jurisdictional claims in published maps and institutional affiliations.

\footnotetext{
Open Access This article is licensed under a Creative Commons Attribution 4.0 International License, which permits use, sharing, adaptation, distribution and reproduction in any medium or format, as long as you give appropriate credit to the original author(s) and the source, provide a link to the Creative Commons license, and indicate if changes were made. The images or other third party material in this article are included in the article's Creative Commons license, unless indicated otherwise in a credit line to the material. If material is not included in the article's Creative Commons license and your intended use is not permitted by statutory regulation or exceeds the permitted use, you will need to obtain permission directly from the copyright holder. To view a copy of this license, visit http://creativecommons.org/ licenses/by/4.0/
}

(c) The Author(s) 2018 